\title{
Mannitol induces selective astroglial death in the CA1 region of the rat hippocampus following status epilepticus
}

\author{
Ah-Reum Ko \& Tae-Cheon Kang ${ }^{*}$ \\ Department of Anatomy and Neurobiology, Institute of Epilepsy Research, College of Medicine, Hallym University, Chunchon 24252, \\ Korea
}

In the present study, we addressed the question of whether treatment with mannitol, an osmotic diuretic, affects astrogliovascular responses to status epilepticus (SE). In saline-treated animals, astrocytes exhibited reactive astrogliosis in the CA1-3 regions 2-4 days after $\mathrm{SE}$. In the mannitol-treated animals, a large astroglial empty zone was observed in the CA1 region 2 days after SE. This astroglial loss was unrelated to vasogenic edema formation. There was no difference in SE-induced neuronal loss between saline- and mannitol-treated animals. Furthermore, mannitol treatment did not affect astroglial loss and vasogenic edema formation in the dentate gyrus and the piriform cortex. These findings suggest that mannitol treatment induces selective astroglial loss in the CA1 region independent of vasogenic edema formation following SE. These findings support the hypothesis that the susceptibility of astrocytes to SE is most likely due to the distinctive heterogeneity of astrocytes independent of hemodynamics. [BMB Reports 2015; 48(9): 507-512]

\section{INTRODUCTION}

The blood-brain barrier (BBB) maintains the brain microenvironment to ensure proper nervous system functions by segregating the systemic environment (1). The BBB is formed by the endothelial cells lining the blood vessels, astrocytic endfeet surrounding the blood vessels, and pericytes embedded in the basement membranes between the endothelial cells and the astrocytes. Astrocytes envelop $>99 \%$ of the BBB endothelium, and play an important role in inducing and maintaining BBB (1-5). BBB disruption leads to the development of various neurological diseases including epilepsy (2-4). BBB disruption

*Corresponding author. Tel: +82-33-248-2524; Fax: +82-33-2482525; E-mail: tckang@hallym.ac.kr

http://dx.doi.org/10.5483/BMBRep.2015.48.9.013

Received 22 January 2015, Revised 10 February 2015, Accepted 16 February 2015

Keywords: Astroglial death, Blood-brain barrier, Epilepsy, Mannitol, Status epilepticus is associated with endothelial damage and reduction in the number of astroglial endfeet attached to microvasular surfaces $(5,6)$.

Status epilepticus (SE), defined as prolonged seizure activity, causes vasogenic edema and astroglial loss (7-14). Although astrocytes are regarded as simple supporting cells with homogenous properties, SE-induced astroglial death shows regional specific patterns. Briefly, acute astroglial death is observed in the molecular layer of the dentate gyrus and the PC before or after neuronal death. In contrast, chronic astroglial death is detected in the CA1 region of the hippocampus. This susceptibility of astrocytes to SE is most likely due to the distinctive anatomical and physiological characteristics of astrocytes independent of hemodynamics (15).

Mannitol, one of the sugar alcohols, is clinically used as an osmotic diuretic to remove excess water from the extra- and intracellular space. In particular, mannitol treatment is the first drug of choice for the treatment of acute brain edema to prevent brain injury due to high intracranial osmotic pressure (4). However, the efficiency of mannitol as an anti-edema agent against vasogenic edema induced by SE is debated. Therefore, elucidating the effect of mannitol treatment on BBB disruption and astroglial loss induced by SE may be noteworthy to understand the pathophysiology of epilepsy. Furthermore, the astroglial response to mannitol may be indicative of the regional specific heterogeneity of astrocytes following SE. During the course of this study, we addressed the question of whether mannitol treatment affects the astroglio-vascular responses to SE.

\section{RESULTS}

Mannitol treatment induces selective astroglial death in the stratum radiatum in the $\mathrm{CA} 1$ region following $\mathrm{SE}$

In saline-treated animals, astrocytes showed typical reactive astrogliosis in the CA1-3 regions 2-4 days after SE. Astrocytic processes became unevenly thick and developed ragged edges during this period (Fig. 1). In these regions, there was no difference in the number of astrocytes compared to non-SE animals (Fig. 1). In the mannitol-treated animals, a large astroglial empty zone $\left(0.4 \pm 0.25 \mathrm{~mm}^{3}\right)$ was observed in the stratum radiatum of the CA1 region 2 days after SE (Fig. 1). Three to four days after $\mathrm{SE}$, the astrocyte-depleted zone in the stratum radia- 


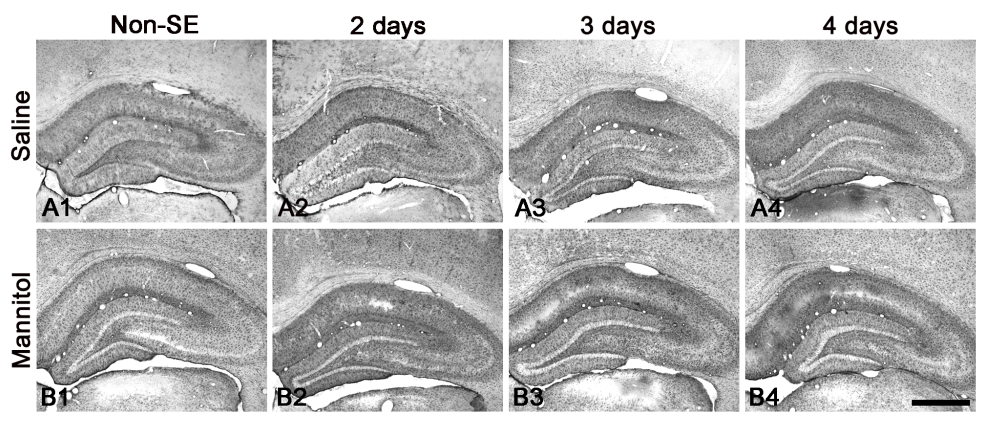

C
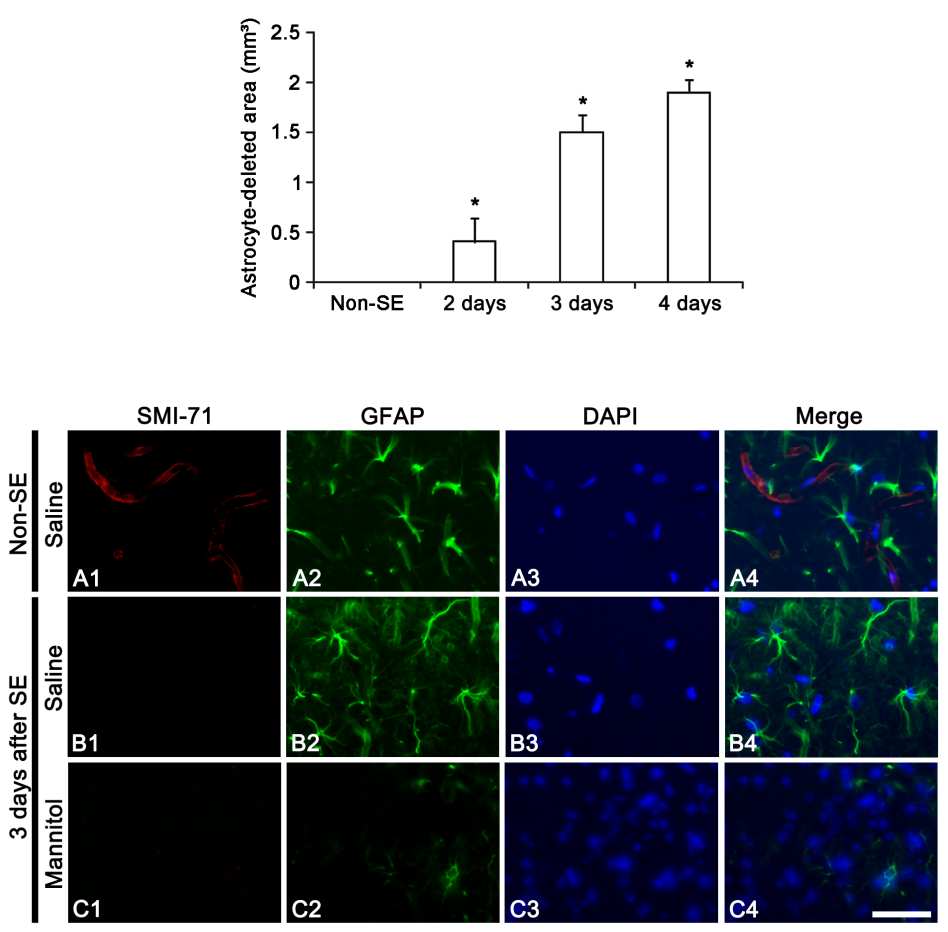

D

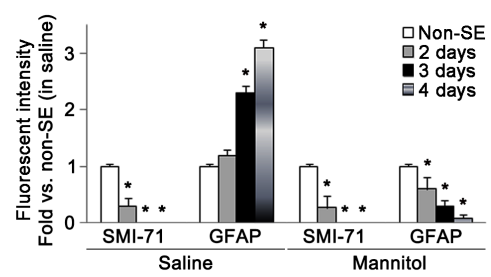

Fig. 1. The effect of mannitol treatment on SE-induced astroglial loss in the hippocampus. Compared to saline (A), mannitol induces a large astroglial empty zone in the stratum radiatum of the CA1 region following SE (B). Bar $=400 \mu \mathrm{m}$. (C) Quantitative analyses of the volume of astrocyte-depleted areas in the hippocampus following SE (mean \pm SD). Significant differences from non-SE animals, ${ }^{*} \mathrm{P}<0.05$.

Fig. 2. The effect of mannitol treatment on SMl-71 and GFAP expression in the CA1 region following SE. Compared to saline (A), Three days after SE, SMI-71 expression gradually decreased in the CA1 region (B). Compared to saline (B), mannitol treatment results in astroglial loss in the CA1 region (C). However, mannitol does not affect loss of SMl-71 immunoreactivity following SE. Bar $=12.5 \mu \mathrm{m}$. (D) Quantitative values (mean \pm S.E.M) of SMI-71 and GFAP fluorescent intensity in the hippocampus. Significant differences from non-SE animals, $* P<0.05$ tum of the CA 1 region was more pronounced $(1.5 \pm 0.17$ and $1.9 \pm 0.13 \mathrm{~mm}^{3}$, respectively), indicating disruption of the astrocyte network (Fig. 1). In addition, the cell bodies of astrocytes were shrunken around the astrocyte-depleted lesion compared to the saline-treated animals (Fig. 1). These findings indicate that mannitol may induce selective astroglial loss in the stratum radiatum of the CA1 region following SE.

\section{Mannitol-mediated astroglial loss is not correlated with vasogenic edema in the hippocampus}

Following SE, SMI-71 (an intact BBB marker) expression decreased gradually in the hippocampus. There was no difference in the reduction of SMI-71 expression between salineand mannitol-treated animals (Fig. 2). However, astroglial loss was observed in the CA1 region of the mannitol-treated ani- 


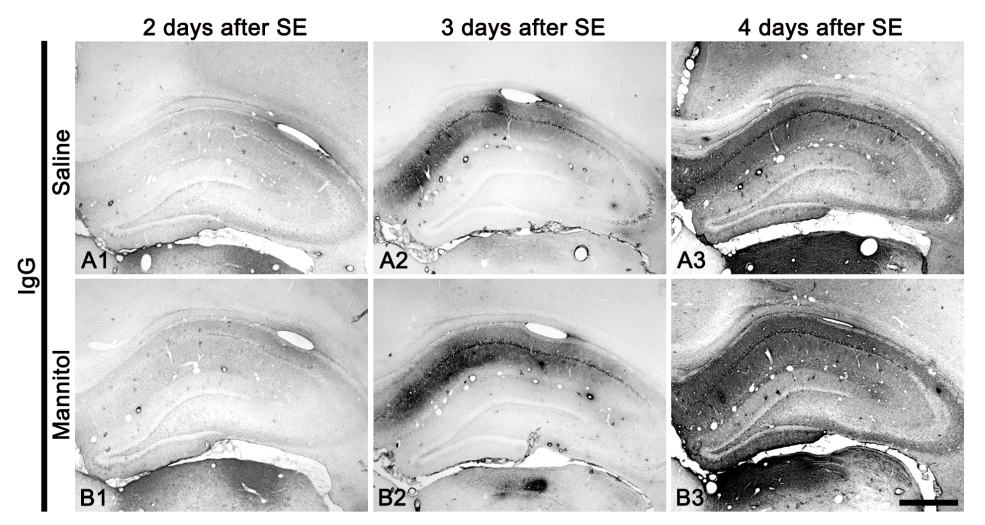

C

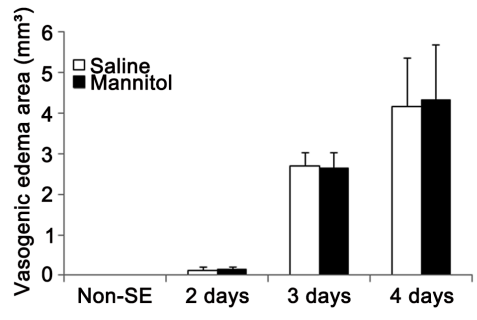

A

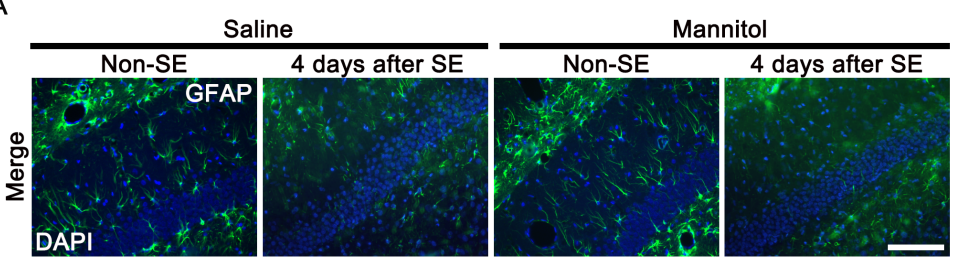

B

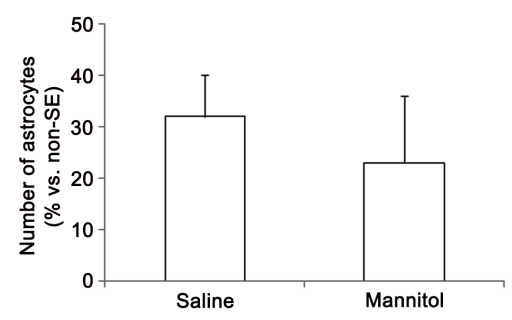

Fig. 3. The effect of mannitol treatment on vasogenic edema in the hippocampus following SE. (A) In saline-treated animals, vasogenic edema is spread to all the hippocampal regions at 4 days after SE. (B) In mannitol-treated animals, the profile of vasogenic edema induced by $\mathrm{SE}$ is similar to that in the saline-treated animals. Bar $=200 \mu \mathrm{m}$. (C) Quantitative analyses of volume of vasogenic edema areas in the hippocampus of following SE (mean \pm SD). mals, whereas it was undetectable in the saline-treated animals (Fig. 2). These findings indicate that mannitol may not affect BBB disruption induced by SE. Therefore, it is likely that astroglial death in the $\mathrm{CA} 1$ region may not be related to $\mathrm{BBB}$ disruption. Furthermore, vasogenic edema was observed in the stratum radiatum, stratum pyramidale and stratum oriens of the CA1 region of saline-treated animals 2-3 days after SE. Vasogenic edema spread to all hippocampal regions 4 days after SE (Fig. 3). In the mannitol-treated animals, the profile of vasogenic edema induced by SE was similar to that observed in the
Fig. 4. The effect of mannitol treatment on astroglial loss in the molecular layer of the dentate gyrus. (A) Four days after SE, astroglial loss is detected in the molecular layer of the dentate gyrus of both saline- and mannitoltreated animals. There is no difference in neuronal loss induced by SE in the hippocampus between saline- and mannitol-treated animals. Bar $=50 \mu \mathrm{m}$. (B) Quantitative analyses of the astroglial death following SE (mean \pm S.D.). saline-treated animals (Fig. 3). However, astroglial loss was detected only in the stratum radiatum of the CA1 region (Fig. 1). These findings suggest that astroglial loss induced by mannitol may be unrelated to vasogenic edema formation.

\section{Mannitol treatment does not affect hippocampal neuronal death induced by SE}

Four days after $\mathrm{SE}$, massive neuronal loss was observed in the hippocampus ( $\mathrm{P}<0.05$ vs. non-SE animals, data not shown). In the saline-treated group, the numbers of Fluore-Jade B (FJB) 
positive degenerative neurons per $\mathrm{mm}^{3}$ in CA1-, CA3 pyramidal cells, and dentate hilar neurons were $21,028 \pm 1,789$, $11,314 \pm 1,017$, and $11,413 \pm 3,443$, respectively. In the mannitol-treated animals, the numbers of FJB positive degenerative neurons per $\mathrm{mm}^{3}$ in CA1-, CA3 pyramidal cells, and dentate hilar neurons were $22,471 \pm 2,047,10,946 \pm 2,679$, and $12,769 \pm 2,941$, respectively. There was no difference in neuronal loss induced by SE in the hippocampus between salineand mannitol-treated animals. These findings indicate that mannitol may not affect SE-induced neuronal death in the hippocampus.

\section{Mannitol treatment does not affect astroglial death in the dentate gyrus}

Consistent with our previous studies $(7,10,11)$, astroglial loss was observed in the molecular layer of the dentate gyrus 4 days after SE (Fig. 4). The number of astroglial cells decreased to $32 \%$ of the amount in non-SE induced animals $(P<0.05$ vs. non-SE). In the mannitol-treated animals, the number of astroglial cells decreased to $23 \%$ of the amount in non-SE animals ( $\mathrm{P}<0.05$ vs. non-SE). Therefore, there was no difference in astroglial loss induced by SE within the molecular layer of the dentate gyrus between saline- and mannitol-treated animals.

\section{Mannitol treatment does not affect vasogenic edema and astroglial death in the piriform cortex}

Two-four days after SE, astroglial loss and vasogenic edema were detected in the PC of both saline- and mannitol-treated animals (data not shown). Two-four days after SE, the astrocytedepleted area and vasogenic edema area in the saline-treated animals were $9.6-16.7$ and $11.6-20.7 \mathrm{~mm}^{3}$, respectively. In the mannitol-treated animals, the astrocyte-depleted area and the vasogenic edema area were 10.1-17.3 and 10.1-18.3 $\mathrm{mm}^{3}$, respectively, at the same time points. There was no difference in SE-induced astroglial loss and vasogenic edema formation within the PC between saline- and mannitol-treated animals.

\section{DISCUSSION}

The major findings of the present study are that mannitol treatment induces selective astroglial loss in the CA1 region following $\mathrm{SE}$, and that this astroglial death is not correlated to $\mathrm{BBB}$ disruption or vasogenic edema formation.

Classically, two major types of brain edema exist (16): cytotoxic edema and vasogenic edema. Cytotoxic edema is caused by intracellular water accumulation while the BBB remains intact (17). Astroglial swelling subsequently or simultaneously leads to dysfunction of astroglial membrane permeability and impairment of reuptake of excitatory neurotransmitters or ions by astrocytes (18). In contrast, vasogenic edema results from dysfunction of both endothelial cells and the BBB, followed by accumulation of water in the extracellular spaces (17). SE increases intracellular $\mathrm{Na}^{+}$and $\mathrm{Ca}^{2+}$ concentrations in astrocytes and dysfunction of $\mathrm{BBB}$, resulting in cytotoxic and vasogenic edema, respectively (19). In both edema types, hyperosmotic mannitol treatment is an effective treatment strategy that creates an osmotic gradient to dehydrate the brain structures (20-22). In a kainate model, mannitol effectively prevented SE-induced lesions by reducing cytotoxic edema $(23,24)$. The present study demonstrates that mannitol treatment does not attenuate vasogenic edema formation in the PC and the hippocampus following SE. Consistent with the present data, some clinical studies reported that mannitol treatment fails to reduce vasogenic edema induced by diabetic ketoacidosis (25-28). Unexpectedly, the present study demonstrated that mannitol treatment induced selective astroglial death in the stratum radiatum in the CA1 region following SE, but did not affect SE-induced neuronal death in the hippocampus. In previous studies $(9,10,12,13)$, SE results in slow autophagic astroglial death (clasmatodendrosis) in the CA1 region after 4-6 weeks. This astroglial damage involves extensive swelling and vacuolization of cell bodies as well as disintegrated and beaded processes. In the present study, damaged astrocytes in the CA1 region showed shrinkage of cell bodies, indicating a reversal of the osmotic concentration gradient between astrocytes and the extracellular space. Therefore, our findings indicate that astrocytes in the stratum radiatum of the CA1 region may be more vulnerable to mannitol-mediated hyperosmolarity than astrocytes in other regions.

SE induces TUNEL-positive apoptotic astroglial death in the molecular layer (not the hilus) of the dentate gyrus within the first week $(7,9,10)$. Similarly, the present study showed that astroglial loss occurred in the molecular layer of the dentate gyrus 4 days after SE. However, this astroglial loss was not affected by mannitol treatment. These findings indicate that osmotic stress may not be a cause of apoptotic astroglial death following SE. In contrast, astroglial damage in the PC is correlated to vasogenic edema, since severe serum-protein extravasation was observed in layer III/IV and spread to layer II three days after SE $(10,11)$. Therefore, it is likely that pressure injury or serum-derived molecules due to vasogenic edema evokes astroglial loss in the PC. In the present study, mannitol treatment did not affect astroglial loss and vasogenic edema in the PC. These findings indicate that mannitol treatment may not be effective for attenuating vasogenic edema induced by SE. With no correlation between astroglial loss and vasogenic edema in the CA1 region in response to $\mathrm{SE}$, our findings indicate that PC astrocytes are vulnerable to vasogenic edema-induced pressure or serum-mediated harmful stress, unlike astrocytes in other regions.

In conclusion, to the best of our knowledge, the present study presents, for the first time, novel evidence that mannitol treatment induces selective astroglial death in the CA1 region. Furthermore, mannitol treatment is not effective for attenuating vasogenic edema and neuronal death induced by SE. Therefore, the present study provides evidence suggesting the regional specific heterogeneity of astrocytes, indicating that mannitol may be re-considered as an anti-edema agent for vaso- 
genic edema-related neurological diseases.

\section{MATERIALS AND METHODS}

\section{Experimental animals and chemicals}

Adult male Sprague-Dawley (SD) rats (7-week old) were used. All the experiments were in strict accordance with the recommendations in the Guide for the Care and Use of Laboratory Animals of the National Institutes of Health (NIH Publications No. 80-23, 1996) and were approved by the Committee on the Ethics of Animal Experiments of the Hallym University (Chunchon, South Korea, Permit Number: 2011-80 \& 2012-62). All reagents were obtained from Sigma-Aldrich (St. Louis, MO, USA), except as noted.

\section{Seizure induction and mannitol treatment}

Animals were given $\mathrm{LiCl}(127 \mathrm{mg} / \mathrm{kg}$ i.p.) $20 \mathrm{~h}$ before pilocarpine treatment. Animals were treated with pilocarpine (25 $\mathrm{mg} / \mathrm{kg}$, i.p.) $30 \mathrm{~min}$ after scopolamine butylbromide $(2 \mathrm{mg} / \mathrm{kg}$, i.p.). Diazepam (Valium, Hoffman la Roche, France; $10 \mathrm{mg} / \mathrm{kg}$, i.p.) was used to quell seizure activity $2 \mathrm{~h}$ after the onset of SE and re-administered as needed. Non-SE animals received saline in place of pilocarpine. Two hours after diazepam treatment, animals were given saline or D-mannitol (30 mg/kg, i.p.) every 24 hr for 4 days.

\section{Tissue processing}

At designated time points (Non-SE, 2 day, 3 days and 4days after $\mathrm{SE}, \mathrm{n}=7$, respectively), animals were perfused transcardially with phosphate-buffered saline (PBS) followed by $4 \%$ paraformaldehyde in $0.1 \mathrm{M} \mathrm{PB}(\mathrm{pH} 7.4)$ under urethane anesthesia $(1.5 \mathrm{~g} / \mathrm{kg}$, i.p.). The brains were postfixed in the same fixative for $4 \mathrm{~h}$ and cryoprotected by immersion in 30\% sucrose-PB at $4^{\circ} \mathrm{C}$ for 2 days. The brains were frozen and sectioned coronally in serial $30 \mu \mathrm{m}$-thick sections. Sections were collected in six-well plates containing phosphate buffered saline (PBS).

\section{Immunohistochemistry}

Sections were incubated with $3 \%$ bovine serum albumin in PBS for $30 \mathrm{~min}$ at room temperature. Sections were then incubated in horse anti-rat immunoglobulin G (IgG) (Vector, USA; diluted 1:250) or mouse anti-GFAP antibody (Chemicon, USA; diluted 1:4,000) in PBS containing $0.3 \%$ triton X-100 overnight at room temperature. Sections were further incubated in secondary antibody and ABC complex (Jackson Immunoresearch Laboratories Inc., USA; diluted 1:250) for $2 \mathrm{~h}$ at room temperature. Thereafter, sections were visualized using 3,3'-diaminobenzidine in $0.1 \mathrm{M}$ Tris buffer. Some sections were incubated in a mixture of mouse anti-GFAP antibody (Chemicon, USA; diluted 1:4,000)/mouse anti-SMI71 antibody (BBB marker; Covance, USA; diluted $1: 1,000)$ in PBS containing $0.3 \%$ triton $\mathrm{X}-100$ overnight at room temperature. Thereafter, sections were incubated in a mixture of FITC-conjugated secondary an- tiserum and Cy3-conjugated secondary antisera (Amersham, USA). The sections were washed three times for 10 min with PBS, and mounted on gelatin-coated slides. For nuclei counterstaining, we used Vectashield mounting medium with DAPI (Vector, USA).

\section{FJB staining}

Sections were rinsed in distilled water, and dried on a slide warmer. The slides were then transferred to $0.06 \%$ potassium permanganate for $15 \mathrm{~min}$ and gently agitated. After rinsing in distilled water for $2 \mathrm{~min}$, the slides were incubated for $30 \mathrm{~min}$ in $0.001 \%$ FJB (Histo-Chem Inc., USA) with gentle shaking in the dark. After rinsing, the slides were dried, dehydrated in $x y$ lene and coverslipped with DPX.

\section{Volumetric analysis and cell counts}

The volume of anti-rat IgG positive region or astrocytes-deleted area was estimated according to a formula based on the modified Cavalieri method: $\mathrm{V}=\Sigma \mathrm{a} \times t_{\text {nom }} \times 1 / \mathrm{ssf}: \mathrm{a}$, area of the

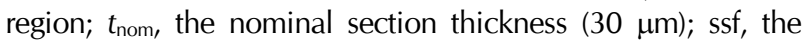
fraction of the sections (1/6 in this study). The subfield areas were delineated with a $2.5 \times$ objective lens $(8,11,14)$. An optical fractionator was used to estimate cell numbers. The number of each cell type $(C)$ in each of the subregions was estimated as: $\mathrm{C}=\Sigma Q^{-} \times \mathrm{t} / \mathrm{h} \times 1 / \mathrm{asf} \times 1 / \mathrm{ssf}: Q^{-}$, the number of cells actually counted; Asf, (a(frame) $/ \mathrm{a}(\mathrm{x}, \mathrm{y}$ step) $\{$ asf $=$ $(\mathrm{a}($ frame $) / \mathrm{a}(\mathrm{x}, \mathrm{y}$ step $))\}$. Cell counts were performed by two different investigators blinded to the classification of tissues.

\section{Quantification of data}

The fluorescence intensity was measured using a computer- assisted image analysis program (The University of Texas ImageTool program V. 3.0 and AxioVision Rel. 4.8 software). Based on the mean range of intensity values, each image was normalized by adjusting the black and white range of the image. Values for background staining were obtained from the corpus callosum. Optical density values were then corrected by subtracting the average values of background noise obtained from 15 image inputs.

\section{Data analysis}

Statistical comparisons were performed by Student t-test or one-way ANOVA test. Bonferroni's test was used for post-hoc comparisons. A P value below 0.05 was chosen as the threshold for significant differences (29).

\section{ACKNOWLEDGEMENTS}

This study was supported by grants from the Healthcare Technology R\&D Project, Ministry for Health, Welfare and Family Affairs (Grant number A111313), the National Research Foundation of Korea (MEST No. 2012R1A2A1A01001775 and 2009-0093812) funded by the Korean government, and Hallym University (HRF-201410-016). 


\section{REFERENCES}

1. Redzic Z (2011) Molecular biology of the blood-brain and the blood-cerebrospinal fluid barriers: similarities and differences. Fluids Barriers CNS 8, 3

2. Kastrup A, Gr schel K, Ringer TM et al (2008) Early disruption of the blood-brain barrier after thrombolytic therapy predicts hemorrhage in patients with acute stroke. Stroke 39, 2385-2387

3. Marik C, Felts PA, Bauer J, Lassmann H and Smith KJ (2007) Lesion genesis in a subset of patients with multiple sclerosis: a role for innate immunity? Brain 130, 2800-2815

4. van Vliet EA, da Costa Araújo S, Redeker S et al (2007) Blood-brain barrier leakage may lead to progression of temporal lobe epilepsy. Brain 130, 521-534

5. Sandoval KE and Witt KA (2008) Blood-brain barrier tight junction permeability and ischemic stroke. Neurobiol Dis 32, 200-219

6. Kwon I, Kim EH, del Zoppo GJ and Heo JH (2009) Ultrastructural and temporal changes of the microvascular basement membrane and astrocyte interface following focal cerebral ischemia. J Neurosci Res 87, 668-676

7. Kang TC, Kim DS, Kwak SE et al (2006) Epileptogenic roles of astroglial death and regeneration in the dentate gyrus of experimental temporal lobe epilepsy. Glia 54, 258-271

8. Kim JE, Choi HC, Song HK, Jo SM and Kim DS (2010) Levetiracetam inhibits interleukin-1 beta inflammatory responses in the hippocampus and piriform cortex of epileptic rats. Neurosci Lett 471, 94-99

9. Kim DS, Kim JE, Kwak SE et al (2008) Spatiotemporal characteristics of astroglial death in the rat hippocampo- entorhinal complex following pilocarpine-induced status epilepticus. Comp Neurol 511, 581-598

10. Kim JE, Ryu HJ, Yeo SI and Kang TC (2011) P2X7 receptor differentially modulates astroglial apoptosis and clasmatodendrosis in the rat brain following status epilepticus. Hippocampus 21, 1318-1333

11. Kim JE, Yeo SI, Ryu HJ et al (2010) Astroglial loss and edema formation in the rat piriform cortex and hippocampus following pilocarpine-induced status epilepticus. J Comp Neurol 518, 4612-4628

12. Ryu HJ, Kim JE, Yeo SI and Kang TC (2011) P65/RelASer529 NF-kB subunit phosphorylation induces autophagic astroglial death (Clasmatodendrosis) following status epilepticus. Cell Mol Neurobiol 31, 1071-1078

13. Ryu HJ, Kim JE, Yeo SI et al (2011) F-actin depolymerization accelerates clasmatodendrosis via activation of lysosome-derived autophagic astroglial death. Brain Res Bull 5, 368-373

14. Sheen SH, Kim JE, Ryu HJ et al (2011) Decrease in dystrophin expression prior to disruption of brain-blood barrier within the rat piriform cortex following status epilepticus. Brain Res 1369, 173-183

15. Kim JE, Kim YJ, Kim JY and Kang TC (2014) PARP1 activation/expression modulates regional-specific neuronal and glial responses to seizure in a hemodynamic-independent manner. Cell Death Dis 5, e1362

16. Klatzo I (1967) Presidental address. Neuropathological aspects of brain edema. I Neuropathol Exp Neurol 26, 1-14

17. Emerson MR, Nelson SR, SamsFvn FE and Pazdernik TL (1999) A global hypoxia preconditioning model: neuroprotection against seizure-induced specific gravity changes (edema) and brain damage in rats. Brain Res Brain Res Protoc 4, 360-366

18. Kimelberg HK, Schools GP, Cai Z and Zhou M (2000) Freshly isolated astrocyte (FIA) preparations: a useful single cell system for studying astrocyte properties. I Neurosci Res 61, 577-587

19. Sperk G (1994) Kainic acid seizures in the rat. Prog Neurobiol 42, 1-32

20. Hartwell RC and Sutton LN (1993) Mannitol, intracranial pressure, and vasogenic edema. Neurosurgery 32, 444-450

21. Vinas FC, Dujovny M and Hodgkinson D (1995) Early hemodynamic changes at the microcirculatory level and effects of mannitol following focal cryogenic injury. Neurol Res 17, 465-468

22. Unterberg AW, Stover J, Kress B and Kiening KL (2004) Edema and brain trauma. Neuroscience 129, 1021-1029

23. Baran $H$, Lassmann $H$, Sperk $G$, Seitelberger $F$ and Hornykiewicz O (1987) Effect of mannitol treatment on brain neurotransmitter markers in kainic acid-induced epilepsy. Neuroscience 21, 679-684

24. Seitelberger F, Lassmann H and Hornykiewicz O (1990) Some mechanisms of brain edema studied in a kainic acid model. Acta Neurobiol Exp (Wars) 50, 263-267

25. Curtis JR, Bohn D and Daneman D (2001) Use of hypertonic saline in the treatment of cerebral edema in diabetic ketoacidosis (DKA). Pediatr Diabetes 2, 191-194

26. Karmat P, Vats A, Gross M and Checchia PA (2003) Use of hypertonic saline for the treatment of altered mental status associated with diabetic ketoacidosis. Pediaric Crit Care Med 4, 239-242

27. Mortazavi MM, Romeo AK, Deep A et al (2012) Hypertonic saline for treating raised intracranial pressure: literature review with meta-analysis. J Neurosurg 116, 210-221

28. Tasker RC and Acerini CL (2014) Cerebral edema in children with diabetic ketoacidosis: vasogenic rather than cellular? Pediatr Diabetes 15, 261-270

29. Kim JE and Kang TC (2011) The P2X7 receptor-pannexin-1 complex decreases muscarinic acetylcholine receptormediated seizure susceptibility in mice. J Clin Invest 121, 2037-2047 